\title{
Design and Analysis of Stimulated Brillouin Scattering in Fiber Optic System for Distributed Sensing Using Optisystem
}

\author{
Reshmi Krishnan G', Gayathri Prasad $S^{2}$ \\ ${ }^{1,2}$ Department of Optoelectronics, University of Kerala, Thiruvananthapuram, Kerala, India
}

\begin{abstract}
Distributed fiber optic sensor based on Brillouin scattering has become increasingly popular due to its advantage of simultaneously measuring temperature and strain. The Stimulated Brillouin Scattering (SBS) threshold, sensing distance, signal-tonoise ratio and spatial resolution are critical parameters to the performance of Brillouin Optical Time Domain Reflectometry (BOTDR sensor. The result had shown SBS suppression by $4 \mathrm{~dB}$ to achieve the maximum sensing distance of up to $60 \mathrm{~km}$ using opti-system. Besides, a 3-bit simplex coding is employed when launching the laser pulses to enhance the SNR at the receiver.
\end{abstract}

Keywords: Stimulated Brillouin Scattering (SBS), Distributed Temperatures Sensing (DTS), Brillouin Optical Time Domain Reflectometry (BOTDR), Power change coefficient, SBS suppression.

\section{Introduction}

A fiber optic sensor uses optical fiber either as the sensing element ("intrinsic sensors"), or as a means of relaying signals from a remote sensor to the electronics that process the signals ("extrinsic sensors"). Depending on the application, fiber may be used because of its small size, or because no electrical power is needed at the remote location, or because many sensors can be multiplexed along the length of a fiber by using light wavelength shift for each sensor, or by sensing the time delay as light passes along the fiber through each sensor. Time delay can be determined using a device such as an optical time-domain reflectometer and wavelength shift can be calculated using an instrument implementing optical frequency domain reflectometry. Compared with other types of sensors, fiber-optic sensors exhibit a number of advantages:

- They consist of electrically insulating materials (no electric cables are required), which makes possible their use e.g. in high-voltage environments.

- They can be safely used in explosive environments, because there is no risk of electrical sparks, even in the case of defects.

- They are immune to electromagnetic interference (EMI), even to nearby lightning strikes, and do not themselves electrically disturb other devices.

- Their materials can be chemically passive, i.e., do not contaminate their surroundings and are not subject to corrosion.

- They have a very wide operating temperature range (much wider than is possible for many electronic devices).

- They have multiplexing capabilities: multiple sensors in a single fiber line can be interrogated with a single optical source

Instead of making measurements at discrete, pre-determined points Distributed Temperature Sensing (DTS) makes continuous measurements over the full length of the optical fibre. As a result DTS is capable of detecting changes in temperature smaller than $0.01^{\circ} \mathrm{C}$ without prior knowledge of where that event might occur. Distributed sensing is also real time - so we get continuous monitoring at all points along the cable at all times. Fast, frequent and accurate measurements of physical factors such as temperature, pressure or strain play a key role when it comes to ensuring the smooth operation of processes in many domestic, commercial and industrial settings. A distributed sensing system is made up of two basic components: the sensor and the detector system. The measurements themselves depend on four variables, or parameters. These include:

- Distance, or range: the distance over which the measurements will be made

- Speed: the time required for each measurement

- Temperature resolution: the size of temperature changes that will be detected

- Spatial resolution: the smallest distance over which a change in temperature can be detected.

The trade-off between these variables determines the performance of the measuring system, and the choice of parameters usually depends on the nature of the application. OTDR is the technique in which an optical pulse is launched in the fiber \& the variation of returned back scattered intensity is monitored so that the variation in fiber scattering coefficient or attenuation along its length can be measured. In sensor, these are used to detect localized measurandinduced variations in the loss or scattering coeff of a continuous sensing fiber. Brillouin scattering is an effect caused by the $\chi^{(3)}$ nonlinearity of a medium, specifically by that part of the nonlinearity which is related to acoustic phonons. For intense beams (e.g. laser light) travelling in a medium such as an optical fiber, the variations in the electric field of the beam itself may produce acoustic vibrations in the medium via electrostriction or radiation pressure. The nonlinear scattering processes cause disproportion attenuation at high optical power levels. It also causes the transfer of optical power from one mode to other modes in forward or backward direction at different frequency. In fact the stimulated scattering mechanisms (SBS or SRS) also provide optical gain but with a shift in frequency. 


\section{International Journal of Science and Research (IJSR)}

ISSN (Online): 2319-7064

Index Copernicus Value (2013): 6.14 | Impact Factor (2014): 5.611

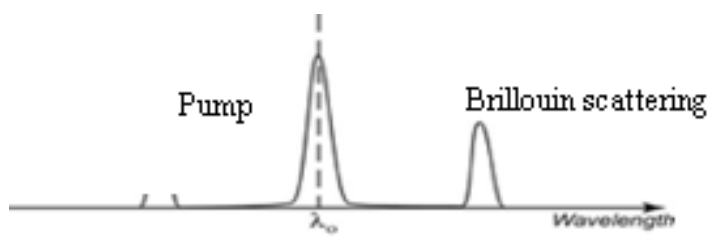

Figure 1: Brillouin scattering

The beam may undergo Brillouin scattering from these vibrations, usually in opposite direction to the incoming beam, a phenomenon known as stimulated Brillouin scattering (SBS).

$$
P_{t h} \approx \frac{21 A_{e f f}}{g_{B} L_{e f f}}
$$

where, $g_{B}$ is $5 \times 10-11 \mathrm{~m} / \mathrm{W}$ and $P_{t h}$ is the threshold power and $\mathrm{g}_{\mathrm{B}}$ is the Brillouin gain constant.

\subsection{Contrast with Rayleigh and Raman Scattering}

- Rayleigh scattering considers only random and incoherent thermal fluctuations, in contrast with the correlated, periodic fluctuations (phonons) that cause the Brillouin scattering.

- The Brillouin scattering occurs due to phonon of acoustic frequency \& a scattered photon. The Raman scattering is due to molecular vibrations.

- In SBS frequency shift maximum in backward direction $\&$ reduces zero in forward direction. In SRS frequency shift maximum.

- SRS optical power threshold up to three orders of magnitude higher than SBS.

- The scattered light is shifted in frequency by about $10 \mathrm{GHz}$ for SBS but by $13 \mathrm{THz}$ for SRS.

- The Brillouin gain bandwidth is extremely narrow in comparison of Raman gain bandwidth.

\subsection{SBS Suppression Factors}

Fiber optic nonlinearity, SBS is suppressed to increase the threshold level. When the SBS threshold is increased, higher power can be launched into the sensing fiber and thus, a greater sensing distance can be achieved. 2.2. SBS suppression factors. In general, the SBS threshold can be evaluated approximatelyusing the following equation:

$$
\mathrm{P}_{\mathrm{SBS}} \approx 21 \mathrm{~K} \mathrm{~A}_{\mathrm{eff}} / \mathrm{g}_{\mathrm{B}} \mathrm{L}_{\mathrm{eff}} \text {, }
$$

where $\mathrm{P}_{\mathrm{SBS}}$ is the SBS threshold power that we wish to increase, $\mathrm{K}$ is the polarization factor, $\mathrm{A}_{\text {eff }}$ is the effective core area of the sensing fiber and $\mathrm{g}_{\mathrm{B}}$ is the Brillouin gain constant which is about $5 \times 10^{11} \mathrm{~m} / \mathrm{W}$ for fused silica glass. $L_{\text {eff }}$ is the effective length which is given by the following equation

$$
\mathrm{L}_{\text {eff }}=\left(1-\mathrm{e}^{-\alpha \mathrm{L}}\right) / \alpha
$$

where a is the fiber attenuation coefficient and $\mathrm{L}$ is the length of the fiber.

Thus the SBS threshold power, $\mathrm{P}_{\mathrm{SBS}}$ depends on the following:

- Polarization factor, $\mathrm{K}$.

- Effective core area, $\mathrm{A}_{\text {eff. }}$

- Brillouin gain coefficient, $g_{B}$.

- Length of fiber, L.

- Effective length of fiber, $\mathrm{L}_{\mathrm{eff}}$.
- Fiber attenuation coefficient, a.

It is found that one of the fiber optic nonlinear effects, namely the Stimulated Brillouin Scattering (SBS) can greatly degrade the performance of the sensor in terms of sensing range and backscattered power level.

\subsection{SBS Suppression Techniques}

Since SBS is identified as the major nonlinearity affecting the sensor performance, various SBS suppression techniques are studied. There are variations of SBS threshold definition. These definitions will be investigated theoretically and through the use of simulation software. The best definition will be employed by the constructed sensor to represent an accurate interpretation of the SBS threshold value. Evaluation of SBS threshold is critical to the accuracy of the simulation result because a comparison of before and after SBS threshold value is needed. Literature review is carried out to verify and to choose the most accurate SBS threshold definition that correctly describes the simulation result. Simplex coding is demonstrated to show the signal-to-noise ratio enhancement of the Brillouin sensor. Besides, increasing the SNR at the receiver of the sensor, Simplex Coding effectiveness in suppressing the SBS threshold power will be investigated theoretically and through simulations. SBS threshold power is increased by employing a 3-bit simplex coding.

\section{Experimental Set-Up}

The optical spectrum analyzer is used to plot the backscattered power versus the backscattered frequency. Comparing to the propagated power, the backscattered power is rather low due to the fact that the backscattered signal in spontaneous Brillouin frequency is extremely weak.
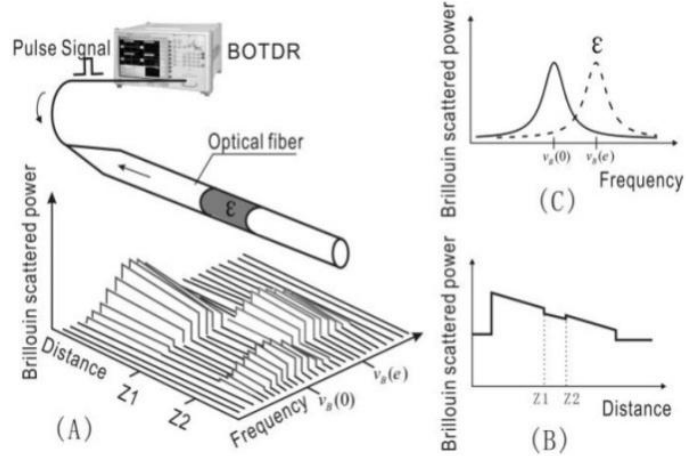

Figure 2: Experimental setup and change in Brillouin scattered power with respect to distance and frequency

The source frequency is compared to the backscattered frequency: The propagated signal is $193.1 \mathrm{THz}$ and as shown below and the backscattered frequency is $193.089 \mathrm{THz}$. Thus the frequency Shift is $193.1 \mathrm{THz}-193.089 \mathrm{THz}=11 \mathrm{GHz}$. $11 \mathrm{GHz}$ is equivalent to the Brillouin Frequency Shift. Thus, the simulated result had accurately shown the effect of Brillouin scattering on frequency shift and power level.

The constructed BOTDR sensor in simulation. 


\section{International Journal of Science and Research (IJSR) \\ ISSN (Online): 2319-7064}

Index Copernicus Value (2013): 6.14 | Impact Factor (2014): 5.611

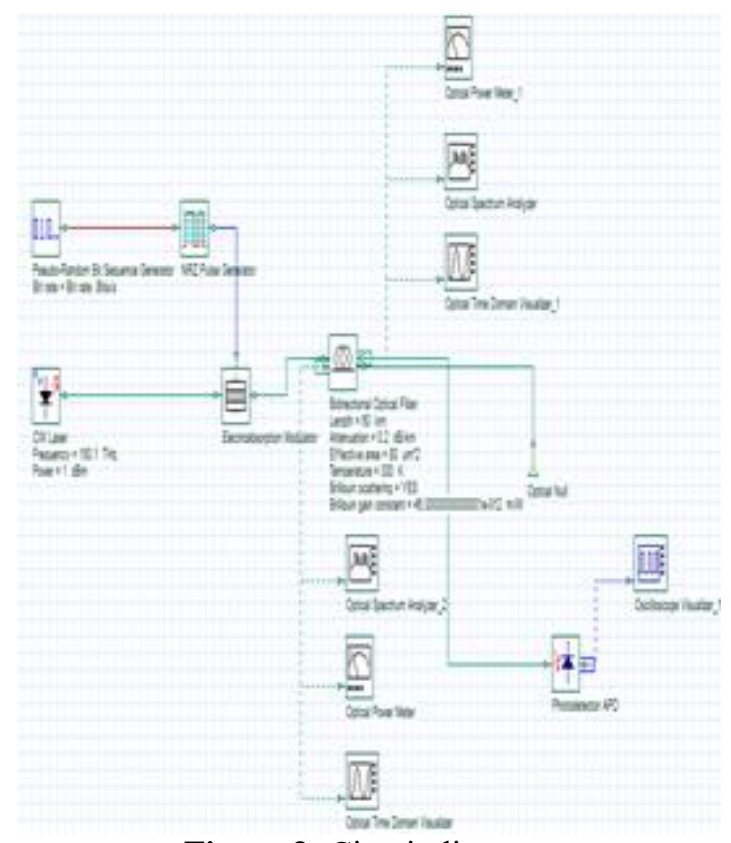

Figure 3: Circuit diagram

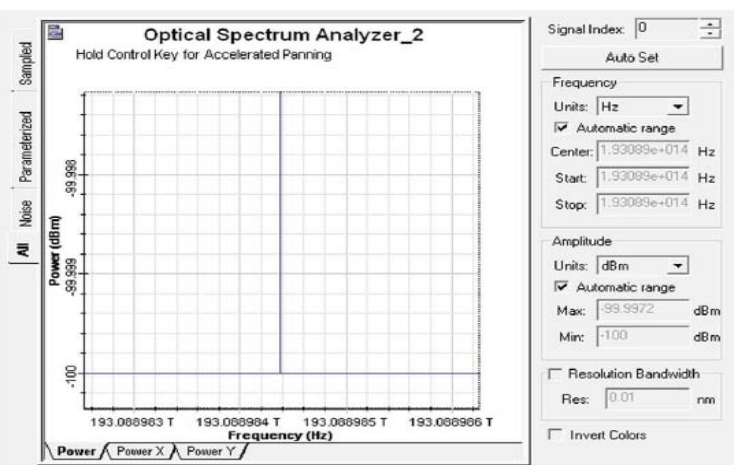

Figure 4: Optical spectrum analyzer output of Brillouin scattering.

The SBS threshold is determined as the input power where the Brillouin Power Change Coefficient starts to degrade. A more suitable definition of SBS threshold would be the input power for which the Brillouin backscatter is equal to the Rayleigh backscatter at the input face. Backscattered power with value higher than the Rayleigh backscatter is considered as the Stimulated backscattered power. To obtain the correct Rayleigh backscattering power level, the Rayleigh backscatter coefficient is 0.0004786 , calculated from the Rayleigh backscattering reflectance. The SBS threshold level is defined at the intersection between the Brillouin backscatter power and the Rayleigh backscatter power. Thus, the SBS threshold power is defined as the input power for which the Brillouin backscatter is equal to the Rayleigh backscatter at the input face. The value of the SBS threshold power for the initial configuration of the sensor is $0 \mathrm{dBm}$. The powers percent change are calculated using the backscattered power of temperature $300 \mathrm{~K}$ which is used as the reference temperature. The reference temperature can be of any desired values as long as it is fixed throughout the calculation of the Brillouin Power Change Coefficient.

To enhance the temperature resolution and the strain resolution of the BOTDR sensor, it is essential to enhance the signal-to noise ratio (SNR) at the receiver. The use of Simplex coding which is based on Hadamard transform will further increase the SNR at the receiver, and thus improving the temperature and strain resolution. Besides a good SNR gain, simplex coding also allows the suppression of nonlinearity in fiber optic cable. In this case, the dominant fiber optic nonlinearity is the Stimulated Brillouin Scattering (SBS) which happens at only a few milli Watt. Stimulated Brillouin Scattering is suppressed by using simplex coding because a lower pulsed input power is injected into the cable for propagation. Thus, the use of simplex coding not only enhances theSNR at the receiver, but also helps to suppress the Stimulated [Brillouin Scattering.

\section{Optisystem}

Optisystem is a CAD environmental tool developed by Optiwave for drawing and simulating the schematics of the WDM, TDM, and DWDM based design models. Optisystem designs are block-based designs, which are interconnected with the wires. The tool is useful in analyzing nonlinear effects with respect to dispersion, noise, jitter etc. In Optisystem, data signal is carried out as a block of data between the blocks of the design transmitted between the blocks. Advantage is easy switching between time domain and frequency domain with the data sent as a block between the optical design models. Performance analysis is done by using eye diagram, BER and Q factor parameters and tools used in Optisystem tool are Spectrum analyzer, Eye Diagram Analyzer and BER analyzer.

\section{Result and Analysis}

\subsection{Input Power Versus Optical Power}

The Back scattered power increases with increasing input power. The threshold SBS power is calculated from the point where the transmitted power intersects with backscattered power. From the graph $\mathrm{P}_{\mathrm{SBS}}=6 \mathrm{~dB}$.

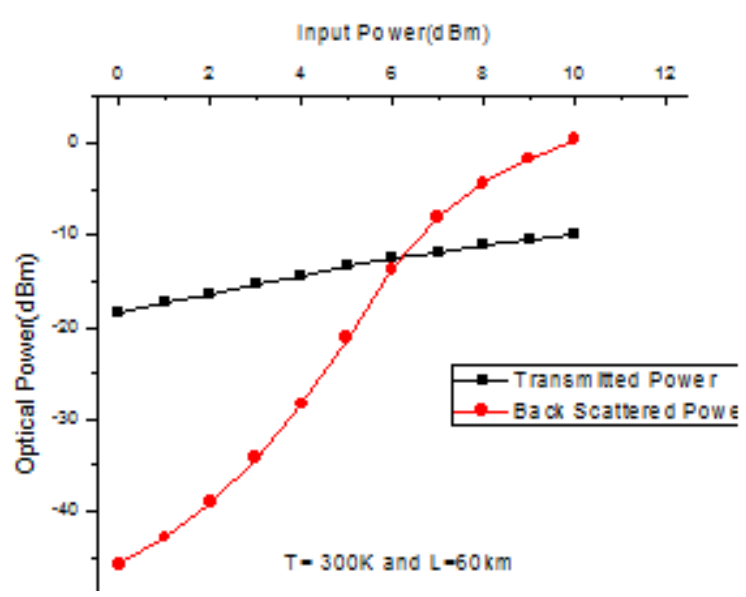

Figure 5: Variation of optical power with respect to the input power

\subsection{Temperature Sensing Accuracy}

The value of Brillouin Power Change Coefficient (\%K) can be obtained from the slope of the fitted line. As shown, the Brillouin Power Change Coefficient or the sensing accuracy is $8.022 \% / \mathrm{K}$. 
International Journal of Science and Research (IJSR)

ISSN (Online): 2319-7064

Index Copernicus Value (2013): 6.14 | Impact Factor (2014): 5.611

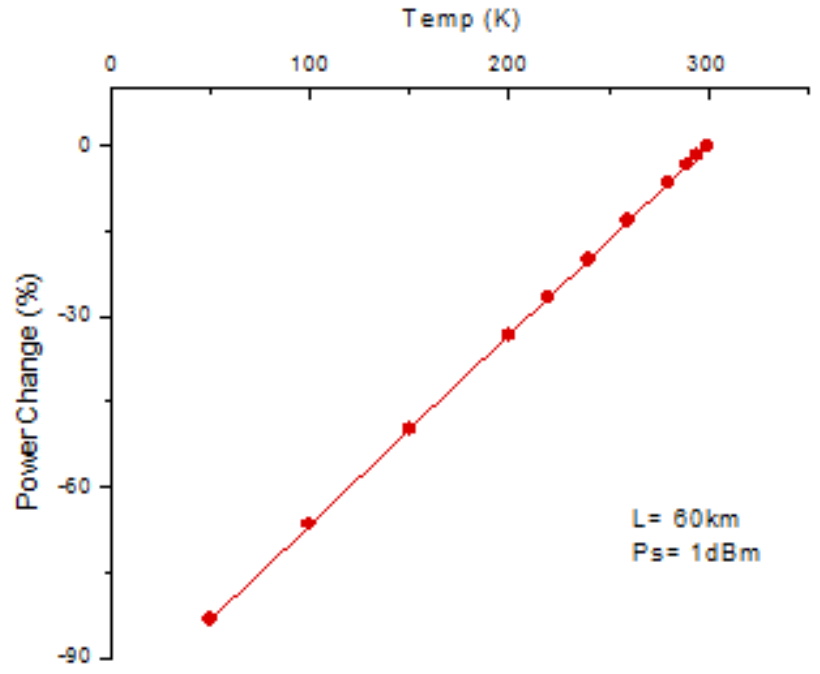

Figure 6: Variation of power change with respect to temperature.

\subsection{Length versus Optical power}

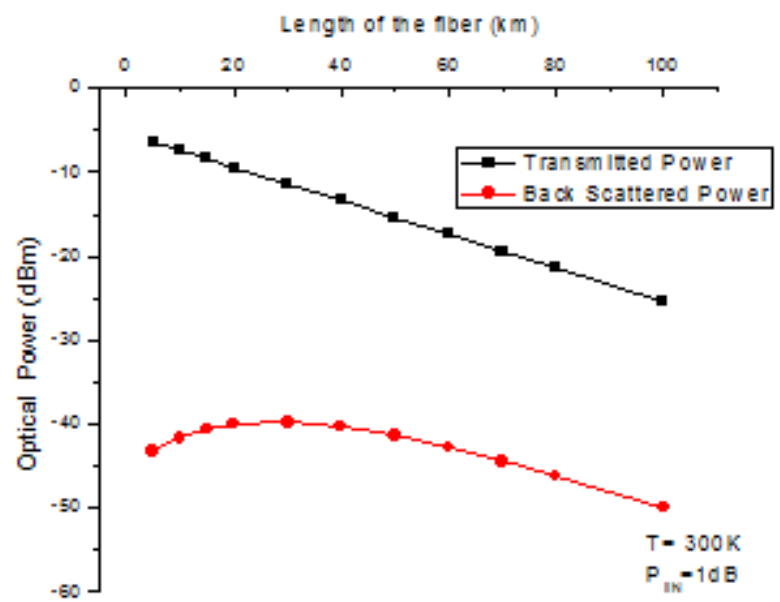

Figure 7: Variation of optical power with respect to length of the fiber.

The value of Transmitted power in $\mathrm{dB}$ decreases with increasing length. But the back scattered power increases up to a limit of $60 \mathrm{~km}$ length and after that it goes on decreasing slowly. Hence, maximum sensing length of a BOTDR can be specified in the range of $60 \mathrm{~km}$.

\subsection{SBS Threshold Definition}

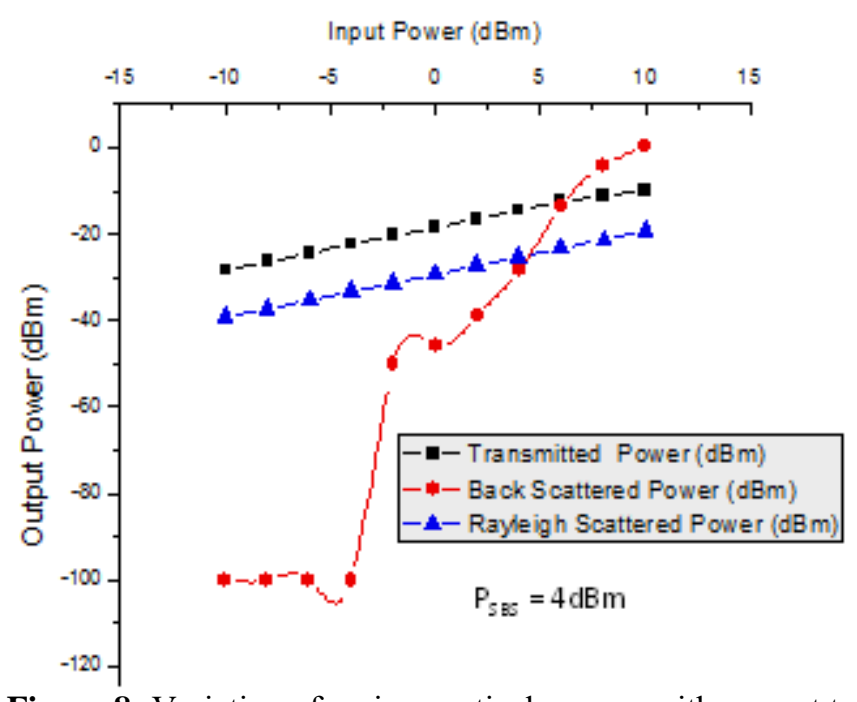

Figure 8: Variation of various optical powers with respect to input power.

Backscattered power with value higher than the Rayleigh backscatter is considered as the Stimulated backscattered power. To obtain the correct Rayleigh backscattering power level, the Rayleigh backscatter coefficient is set as 0.0004786. As shown, the SBS threshold level is definedat the intersection between the Brillouin backscatter power and theRayleigh backscatter power.Thus, the SBS threshold power is defined as the input power forwhich the Brillouin backscatter is equal to the Rayleigh backscatterat the input face. The value of the SBS threshold power for the initial configuration of the sensor is $4 \mathrm{dBm}$.

\subsection{Simplex Coding}

Before SBS Suppression

Using Pseudo Random Bit sequence generator ( $\left.2 \mathrm{e}^{9} \mathrm{bits} / \mathrm{s}\right)$

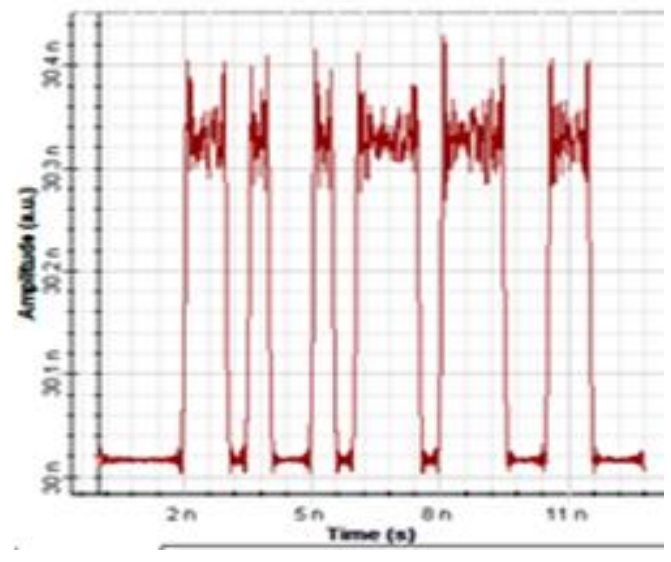




\section{International Journal of Science and Research (IJSR) \\ ISSN (Online): 2319-7064}

Index Copernicus Value (2013): 6.14 | Impact Factor (2014): 5.611

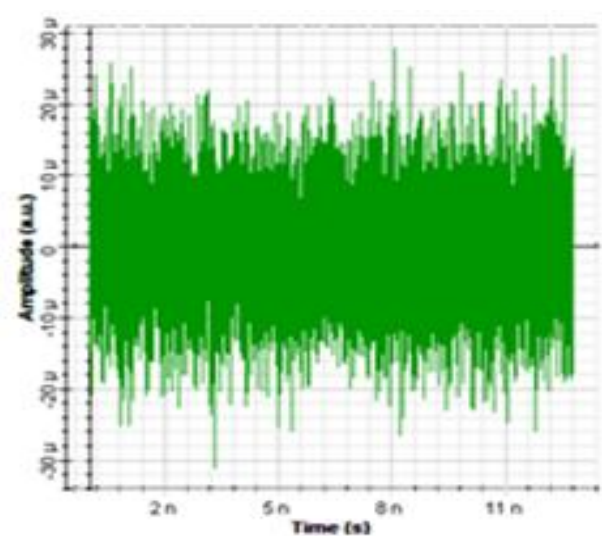

Figure 9: Signal output of PRBS before SBS Suppression.

Using User Defined Bit Sequence Generator (2 $\mathrm{e}^{9} \mathrm{bits} / \mathrm{s} \&$ Code used:110)
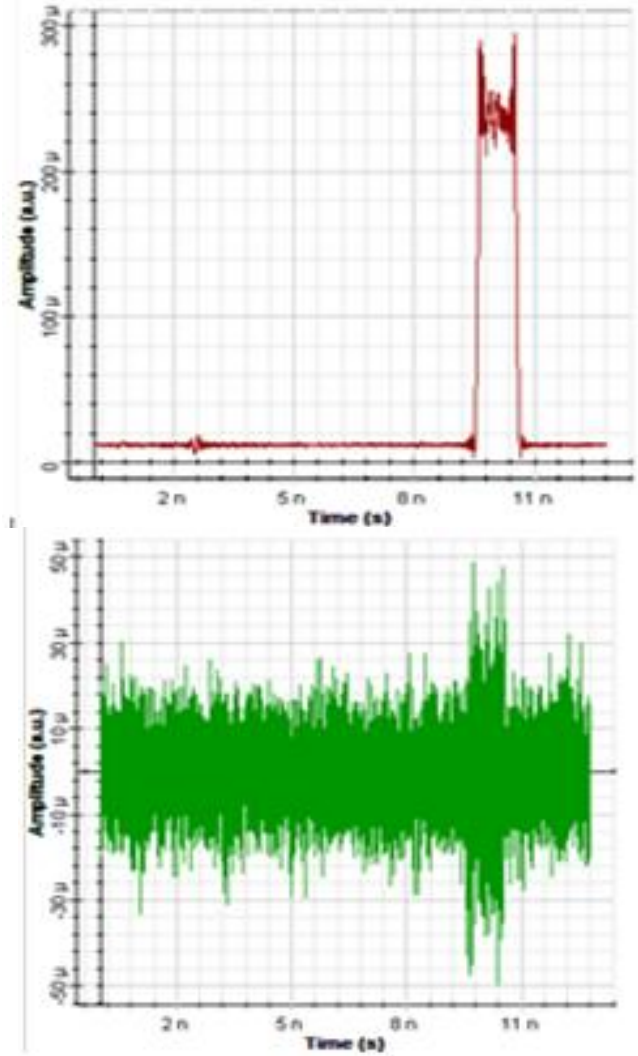

Figure 10: Signal output of PRBS using user defined bit sequence before SBS suppression.

After SBS suppression

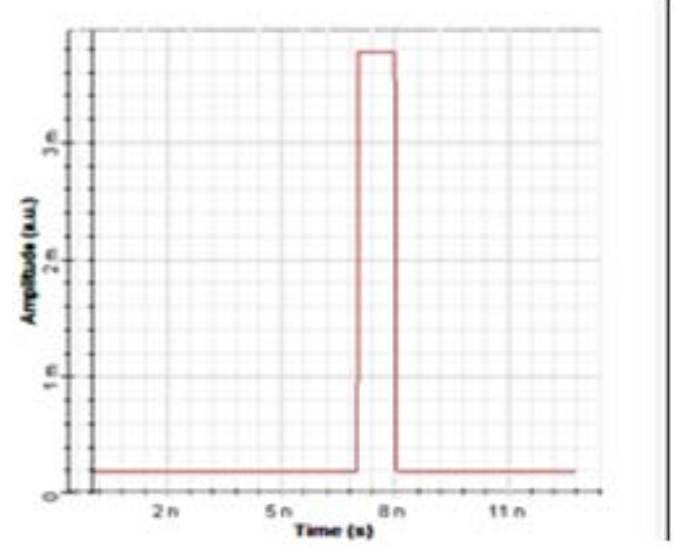

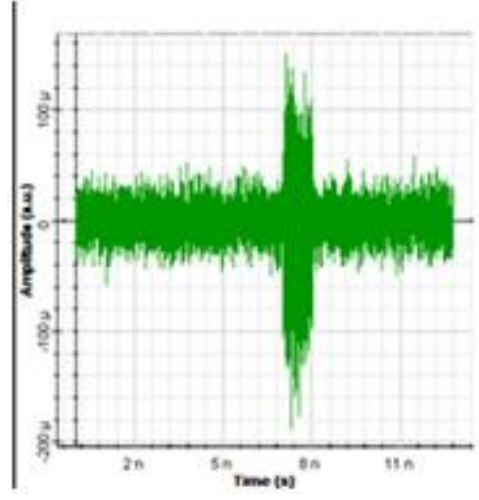

Figure 11: Signal output before and after SBS Suppression using user defined bit sequence generator

From figures under the SIMPLEX CODING,

Using Pseudo-Random Bit sequence Generator:

Noise level at $8 \mathrm{~ns}-2.04 \mathrm{e}-5$

Using User-defined Bit Sequence Generator:

Before Suppression:

Noise level at 8 ns- 7.06e-6

After Suppression:

Noise Level at 8ns- $-2.78 \mathrm{e}-6$

By using Simplex Coding noise level SBS can be suppressed and there by SNR increases.

\section{Conclusion}

This paper has presented the application of temperature sensing using spontaneous Brillouin backscattering based fiber optic sensor. Brillouin Optical Time Domain Reflectometry (BOTDR) utilizes spontaneous Brillouin scattering, which has a weak backscatter power level. Sophisticated detection method must be used to detect the weak reflection signal. Thus, the suppression of Stimulated Brillouin Scattering becomes mandatory and important. Work has been done to increase the SBS threshold power to achieve a higher detectable power, longer sensing range, less equipment cost and higher sensing accuracy. The proposed improvement of parameters to increase the SBS threshold power can be achieved by fabricating the SBS suppression fiber using the Vapor phase Axial Deposition (VAD) method. VAD is a method of fabricating graded-index optical fibers in which fine glass particles of silicon dioxide and germaniumdioxide are synthesized and deposited. The SBS threshold is increased due to the longitudinal change in the dopant concentration of the core and cladding. When the dopant content is changed, theBrillouin frequency shift changes along the fiber and this in turn resultsin a wide SBS gain bandwidth. Thus, the proposed improvement of parameters can be implemented. With the SBS threshold increased, pre-amplification of pulses before launching into fiber can be eliminated. Thus, the cost of amplifier, EDFA can be saved.

\section{References}

[1] Yan, Si Zhi, and Lee Sheng Chyan. "Performance enhancement of BOTDR fiber optic sensor for oil and gas pipeline monitoring." Optical Fiber Technology 16.2 (2010): 100-109. 


\section{International Journal of Science and Research (IJSR) \\ ISSN (Online): 2319-7064}

Index Copernicus Value (2013): 6.14 | Impact Factor (2014): 5.611

[2] Inaudi, Daniele, and Branko Glisic. "Integration of distributed strain and temperature sensors in composite coiled tubing." Smart Structures and Materials. International Society for Optics and Photonics, 2006.

[3] Horiguchi, Tsuneo, et al. "Development of a distributed sensing technique using brillouin scattering: Optical fiber sensors." Journal of lightwave technology 13.7 (1995): 1296-1302.

[4] GovindP Agrawal, Nonlinear Fiber Optics, Academic Press, 2001, ISBN 0-12 045143-3.

[5] Shimizu, Tomoya, et al. "Evaluation methods and requirements for the stimulated Brillouin scattering threshold in a single-mode fiber." Optical Fiber Technology 14.1 (2008): 10-15.

[6] Shiraki, Kazuyuki, M. Ohashi, and M. Tateda. "Suppression of stimulated Brillouin scattering in a fibre by changing the core radius." Electronics letters 31.8 (1995): 668-669.

[7] Hansryd, J., et al. "Increase of the SBS threshold in a short highly nonlinear fiber by applying a temperature distribution." Journal of lightwave technology19.11 (2001): 1691 .

[8] Shiraki, Kazuyuki, MasaharuOhashi, and MitsubiroTateda. "SBS threshold of a fiber with a Brillouin frequency shift distribution." Lightwave Technology, Journal of 14.1 (1996): 50-57.

[9] Smith, Richard G. "Optical power handling capacity of low loss optical fibers as determined by stimulated Raman and Brillouin scattering." Applied Optics 11.11 (1972): 2489-2494.

[10] Lopez-Higuera, Jose-Miguel, Luis Rodriguez-Cobo, and AdlfoCobo. "Optical Sensors: A Comprehensive Approach." Optical Sensors. Optical Society of America, 2015.

[11] Maughan, Sally M. Distributed fibre sensing using microwave heterodyne detection of spontaneous Brillouin backscatter. Diss. University of Southampton, 2002.

[12] Shiraki, Kazuyuki, MasaharuOhashi, and Mitsuhiro Tateda. "Performance of strain-free stimulated Brillouin scattering suppression fiber." Lightwave Technology, Journal of 14.4 (1996): 549-554.

[13] Jacobsen, G., and B. Tromborg. "Dynamic stimulated Brillouin scattering analysis." Lightwave Technology, Journal of 18.3 (2000): 416-424.

[14] Wan, Ping, and Jan Conradi. "Impact of double Rayleigh backscatter noise on digital and analog fiber systems." Lightwave Technology, Journal of 14.3 (1996): 288-297.

[15] Sahu, Prasant K., et al. "Analysis of Brillouin-based distributed fiber sensors using optical pulse coding." Optical Fiber Communication Conference. Optical Society of America, 2008.

[16]Lee, Duckey, et al. "Analysis and experimental demonstration of simplex coding technique for SNR enhancement of OTDR." LTIMC-15, Proceedings IEEE LTIMC, New York (2004).

[17] Michael D. Jones, Using simplex codes to improve OTDR sensitivity, IEEE Photon. Technol. Lett. 15 (7) (1993).

[18] Inaudi, Daniele, and BrankoGlisic. "Long-range pipeline monitoring by distributed fiber optic sensing." Journal of pressure vessel technology 132.1 (2010): 011701.
[19]Ohashi, Masaharu, and Mitsuhiro Tateda. "Design of strain-free-fiber with nonuniform dopant concentration for stimulated Brillouin scattering suppression." Lightwave Technology, Journal of 11.12 (1993): 19411945.

\section{Author Profile}

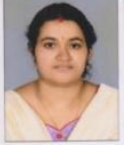

Reshmi Krishnan G received M.Sc in Electronics and Communication from Al-Azhar College of Arts and Science, Thodupuzha (Mahatma Gandhi University) and pursuing M.Tech Degree from Department of Optoelectronics, University of Kerala, Thiruvananthapuram.

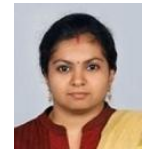

Gayathri Prasad S received B.Tech from $\mathrm{MG}$ College of Engineering and Technology (Cochin University) and pursing M.Tech Degree from Department of Optoelectronics, University of Kerala,Thiruvananthapuram 